\title{
Self-Assembly of Narrowly Distributed Carboxy-Terminated Linear Polystyrene Chains in Water via Microphase Inversion
}

\author{
Shiyong Liu, ${ }^{\dagger}$ Tengjiao Hu, ${ }^{\ddagger}$ Haojun Liang, ${ }^{\ddagger}$ Ming J iang, ${ }^{*, \dagger}$ and $\mathrm{Chi} \mathrm{Wu}^{*, \pm, \$}$ \\ Institute of Macromolecular Science and Laboratory of Molecular E ngineering of Polymers, \\ Fudan University, Shanghai 200433, China; The Open Laboratory of Bond-Selective Chemistry, \\ Department of Chemical Physics, University of Science and Technology of China, Hefei, Anhui, China; \\ and Department of Chemistry, The Chinese University of Hong Kong, Shatin, Hong Kong
}

Received J une 27, 2000; Revised Manuscript Received August 29, 2000

\begin{abstract}
We found by surprise that $\omega$-metal carboxy-terminated linear polystyrene chains could selfassemble into stable surfactant-free colloidal nanoparticles if the polymer solution in THF or DMF is added dropwise into an excess of water. Our results showed that the self-assembly was controlled by competition between the intrachain contraction and interchain association. There is a delicate balance between thermodynamic and kinetics. Inside the self-assembled micelle-like nanostructure, the polymer chains were in a metastable state and kinetically frozen. The self-assembly resembles the initial stage of surfactant-free emulsion polymerization. The size of the nanostructures was essentially governed by the surface area occupied per ionic end group. A variation of the initial conditions, such as the polymer concentration in THF and DMF, solvent, and counterions, can make either the intrachain contraction or interchain association dominant so that the self-assembly can be tailored.
\end{abstract}

\section{Introduction}

Emulsifier-free emulsion polymerization was developed to prepare monodisperse and clean latex particles which are stabilized only by ionic groups located at the chain end, such as sulfate from the initiator potassium persulfate (KPS). ${ }^{1,2}$ It has been suggested that in the initial stage of the particle formation the resultant low molar mass hydrophobic polymer chains with an ionic end group could act as an emulsifier, and these short chains were identified by conductometric titration. ${ }^{3-6}$ Presumably, they formed a micelle-like structure with the ionic end groups located on the surface. It is not hard to imagine that these micellelike structures must be swollen by the hydrophobic monomers presented in the dispersion so that a further reaction inside these micelle-like nanostructures could lead to stable polymeric latex particles. Therefore, it is important for us to understand how these short polystyrene chains are selfassembled in water in the initial polymerization stage.

Our previous studies have demonstrated that a slightly carboxylated commercial styrene-(ethylene-cobutene)-styrene triblock copolymer ionomer and randomly carboxylated or sulfonated polystyrene ionomers can form surfactant-free colloidal nanoparticles stable in water. ${ }^{7-10}$ In these cases, we had no control over the distribution of a few molar percent of ionic groups on the polymer chain backbone. Therefore, in the present study, we decided to investigate the self-assembly of polymer chains with a well-defined chain architecture, namely, narrowly distributed carboxy-terminated linear polystyrene chains. The objectives are (1) to find how these carboxy-termi nated polystyrene chains with only one ionic end group per chain can self-assemble together in water and (2) to provide a model system for the study of the initial stage of surfactant-free emulsion polymerization. We studied how various initial conditions,

† Fudan University.

₹ University of Science and Technology of China.

$\S$ The Chinese University of Hong Kong.

* To whom correspondence should be addressed. such as the ionic end groups, counterions, solvent, polymer concentration, and chain length, could affect the self-assembly.

\section{Experimental Section}

Synthesis of MCPS and DCPS. Anionic polymerization of styrene was carried out at $-20^{\circ} \mathrm{C}$ in a benzene/THF (3/1, $\mathrm{v} / \mathrm{v}$ ) mixture using $\mathrm{n}-\mathrm{BuLi}$ in cyclohexane (about $1 \mathrm{M}$ ) as initiator. The synthesis details can be found elsewhere.11,12 The living polystyryl was terminated by $\mathrm{CO}_{2}$ free of oxygen and protonic impurities. The polymer was recovered by twice precipitation in methanol. The crude product was purified by passing through silicon gel columns monitored by thin-layer chromatography (TLC) using $\mathrm{CH}_{2} \mathrm{Cl}_{2}$ and ethyl ether as eluents, successively. ${ }^{13}$ The ethyl ether elution was concentrated and precipitated into methanol. Such obtained polymer was dried under vacuum at $50{ }^{\circ} \mathrm{C}$. FTIR shows that MCPS has a strong carbonyl stretching peak at $1706 \mathrm{~cm}^{-1} \cdot{ }^{13} \mathrm{C} \mathrm{NMR}$ shows a signal of carbonyl at $179 \mathrm{ppm}$. The functionality of MCPS was calculated from SEC and acid-base titration by using $\mathrm{NaOCH}_{3} /$ methanol/toluene.

In the preparation of DCPS, instead of using dimethyl maleate as an end-capping reagent for the living polystyryl anion, ${ }^{14}$ we used di-tert-butyl maleate. It not only reduced the attacking possibility of polystyryl anion on the ester carbonyl but also made it possible to conduct the hydrolysis under a milder condition. Di-tert-butyl maleate was prepared from isobutylene and maleic acid. Its characterization shows that ${ }^{1} \mathrm{H} N M R\left(\mathrm{CDCl}_{3}\right): \delta 6.05(\mathrm{~s}, 2 \mathrm{H}, \mathrm{CH}=\mathrm{CH})$ and $1.51(\mathrm{~s}, 18 \mathrm{H}$, t-Bu); FTIR: 1720 and $1632 \mathrm{~cm}^{-1}$. The living PSLi was prepared in THF at $-80^{\circ} \mathrm{C}$. The di-tert-butyl maleate solution (twice the amount of n-BuLi used) solution in freshly dried THF was added by syringe. The characteristic dark color of the polystyrl anion changed to light yellow in seconds. De gassed methanol was added to terminate the reaction. The purification of diester-terminated polystyrene was identical to that used for MCPS. FTIR shows an ester carbonyl peak at $1727 \mathrm{~cm}^{-1}$. The diester-terminated polystyrene was hydrolyzed in a mixture of dioxane and $\mathrm{HCl}$ at $80{ }^{\circ} \mathrm{C}$ for $24 \mathrm{~h}$. The resultant polymer was recovered by twice precipitation in methanol and dried under vacuum at $50{ }^{\circ} \mathrm{C}$. FTIR shows a complete disappearance of the ester carbonyl peak at 1727 $\mathrm{cm}^{-1}$, but two new bands at 1712 and $1742 \mathrm{~cm}^{-1}$, which are attributed to carbonyl stretching of the hydrogen-bonded and 
free carboxyls, respectively. The functionality of DCPS was 1.90, close to a theoretical value of 2 .

Preparation of MCPS and DCPS I onomers. The sodium and lithium salts of MCPS and DCPS were prepared by titrating the MCPS and DCPS solution in THF to a phenolphthalein end point with a sodium methylate or lithium methylate tolulene/methanol (90/10, v/v) solution. The zinc salts of MCPS and DCPS were prepared by a new procedure that ensures a complete neutralization in which $30 \%$ excess of zinc acetate dihydrate toluene/methanol $(90 / 10, v / v)$ solution was added into the MCPS solution in THF, and the mixture was refluxed for $3 \mathrm{~h}$ before introducing an appropriate amount of cyclohexane to form azeotrope with acetic acid. All the solvents were removed by a rotary evaporator after the reaction. The excessive zinc acetate was removed by an ultracentrifugation of the zinc ionomer solution in THF. The resultant clear solution was concentrated, precipitated, and vacuum-dried. The nomenclature used for the MCPS or DCPS ionomer is M-MCPS-x or M-DCPS-x, where M designates its counterion, $\mathrm{Li}^{+}, \mathrm{Na}^{+}$, or $\mathrm{Zn}^{++}$, and $\mathrm{x}$ denotes its molar mass.

Microphase Inversion. Each ionomer sample was first dissolved in THF or DMF under a continuous stirring for at least 2 days to ensure a complete dissolution. The initial polymer concentration of the stock solution in THF or DMF was $1.00 \times 10^{-2} \mathrm{~g} / \mathrm{mL}$. The solution was diluted to different concentrations of the stock solution in the range $10^{-2}-10^{-4}$ $\mathrm{g} / \mathrm{mL}$. The resultant ionomer solution in THF or DMF was added dropwise into an excess of water under ultrasonification, resulting in a transparent stable colloidal dispersion in water. The dispersion contains $1 \% \mathrm{THF}$, and the final ionomer concentration is $1 \times 10^{-4} \mathrm{~g} / \mathrm{mL}$ or less. The resultant dispersion was diluted with a THF/water (1/99 v/v) mixture for fluorescence and laser light-scattering (LLS) measurements. The dispersion can also be prepared by adding a large amount of deionized water dropwise into the ionomer solution in THF or DMF.

Fluorescence Measurement. The steady-state fluorescence was measured using a Perkin-EImer Luminescence spectrometer LS50 with a right-angle geometry $\left(90^{\circ}\right.$ collecting optics) and an excitation wavelength of $335 \mathrm{~nm}$. The excitation and emission slits were 5 and $3 \mathrm{~nm}$, respectively. Pyrene with a concentration of $2.0 \times 10^{-6} \mathrm{M}$ was used to probe the selfassembly of the ionomer chains in water. The solutions were ultrasonificated to ensure a complete dispersion of pyrene and stored at room temperature for at least $12 \mathrm{~h}$ before the fluorescence measurements.

Laser Light Scattering. A modified commercial LLS spectrometer (ALV/SP-125) equipped with a multi- $\tau$ digital time correlator (ALV-5000E) and a solid-state laser (Uniphase, output power $\cong 50 \mathrm{~mW}$, at $\lambda_{0}=532 \mathrm{~nm}$ ) was used. The details of LLS instrumentation and theory can be found elsewhere. ${ }^{8}$ All the measurements were done at $25.0 \pm 0.05{ }^{\circ} \mathrm{C}$. The dispersions used in LLS were clarified by a $0.45 \mu \mathrm{m}$ Anotop filter. Static LLS leads to the weight-average molar mass $\left(M_{w}\right)$. The measured time correlation functions were analyzed by both the cumulants and Laplace inversion (CONTIN) programs. For M-MCPS ionomers in THF and in water, the specifical refractive index increments $(\mathrm{dn} / \mathrm{dC})$ are 0.183 and $0.250 \mathrm{~mL} / \mathrm{g}$, respectively.

\section{Results and Discussion}

Figure 1 shows that adding the polymer solution in THF dropwise into an excess of water resulted in narrowly distributed polystyrene nanoparticles in the range $10-70 \mathrm{~nm}$, and the relative distribution width $\left(\mu_{2} /\langle\Gamma\rangle^{2}\right)$ is less than 0.05 . The average particle density $\langle\rho\rangle$ and aggregation number $\left\langle\mathrm{N}_{\text {agg }}\right\rangle$ are $0.069 \mathrm{~g} / \mathrm{cm}^{3}$ and $2.5 \times 10^{3}$, respectively, where $\langle\rho\rangle=M_{w} /\left(N_{A} 4 \pi\left\langle R_{h}\right\rangle^{3} / 3\right)$ and $\left\langle N_{\text {agg }}\right\rangle=M_{w, \text { particle }} / M_{w, \text { chain. }}$ In contrast, the addition of water into the polymer solution resulted in much larger particles with $\langle\rho\rangle=0.093 \mathrm{~g} / \mathrm{cm}^{3}$ and $\left\langle\mathrm{N}_{\text {agg }}\right\rangle=9.3$ $\times 10^{4}$. In the both cases, the resultant particles were very stable, and there was no change in the size distribution over 2 months. Note that the values of $\langle\rho\rangle$

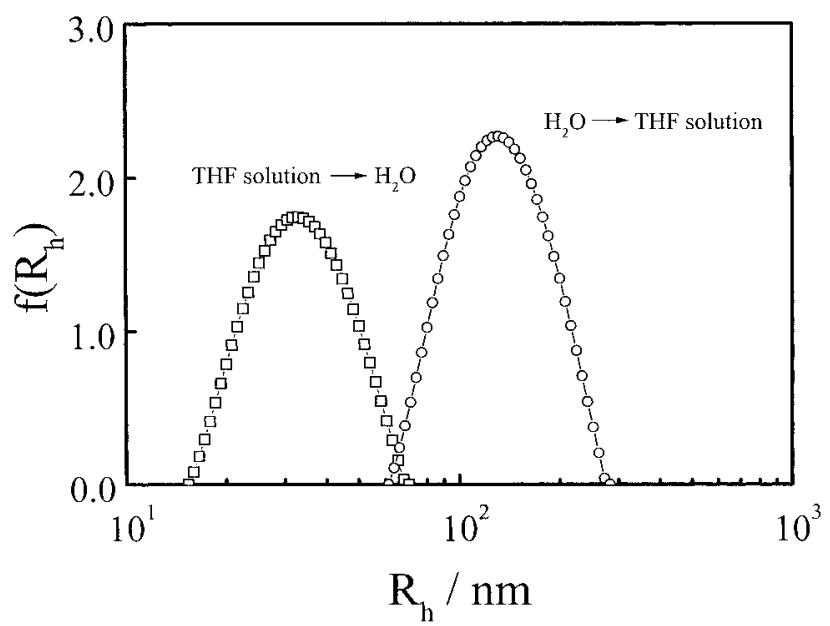

Figure 1. Comparison of number distributions $f\left(R_{h}\right)$ of hydrodynamic radius of self-assembled Na-MCPS-3.9K nanostructures formed after mixing the polymer THF solution and water in two different ways, where the initial polymer concentration in THF was $1.00 \times 10^{-3} \mathrm{~g} / \mathrm{mL}$.

are quite low, indicating that there are a lot of solvent mol ecules trapped inside the collapsed chains. The low values of $\langle\rho\rangle$ are also partially attributed to the use of $\left\langle R_{h}\right\rangle$ in the calculation of the particle volume. It is expected that the particle surface is not smooth so that using $\left\langle R_{h}\right\rangle$ in the calculation would lead to a larger vol ume and a low particle density. Thermodynamically, MCPS should precipitate out in a mixture of water $/ \mathrm{THF}$ (99/1, v/v) because water is a nonsolvent. The stabilization can be qualitatively explained.

In THF, we expected that the ionic end groups were associated together similar to the aggregation of MCPS and DCPS in toluene and cyclohexane. ${ }^{14,15}$ Static LLS measurements showed that Na-MCPS-1.8K and $\mathrm{Zn}$ MCPS-1.8K solutions in THF contain the aggregates made of 2-3 polymer chains. After each drop of the THF solution was added into water, THF immediately mixed with water so that the insoluble MCPS chains must undergo an intrachain contraction and an interchain association, i.e., a fast microphase inversion. The aggregation of the hydrophobic chains forced the ionic end groups to stay on the periphery. Note that each chain has only one ionic group at its end so that the selfassembled nanostructure is similar to a micelle. In the self-assembly, the number of ionic groups per particle $\left(\mathrm{N}_{\text {ionic }}\right)$ equals the number of the chains inside, so that $\mathrm{N}_{\text {ionic }}$ is proportional to the particle volume, i.e., $\mathrm{N}_{\text {ionic }}$ $\propto \mathrm{R}^{3}$ with $\mathrm{R}$ being the particle radius. On the other hand, the particle surface area (S) is proportional to $\mathrm{R}^{2}$. Therefore, the surface area per ionic group (sionic $=$ $S / N_{\text {ionic }} \propto 1 / R$ ) decreases as $R$ increases. For each given system, there should exist a minimum of sionic at which the interchain association stops and the self-assembled nanostructures are stabilized by electrostatic repul sion. Note that the local polymer concentration inside the particle is relatively higher $(\sim 10 \%)$ so that the chain relaxation is expected to be slow, and the interparticle chain fusion should be nearly impossible even when two such particles are colloided with each other. Moreover, the polymer chains and water have a similar density $\left(\sim 1 \mathrm{~g} / \mathrm{cm}^{3}\right)$ so that there should be nearly no sedimentation force.

In the case of adding water into the THF solution, the solvent quality was gradually changed, which leads to a slow interchain association and intrachain contrac- 


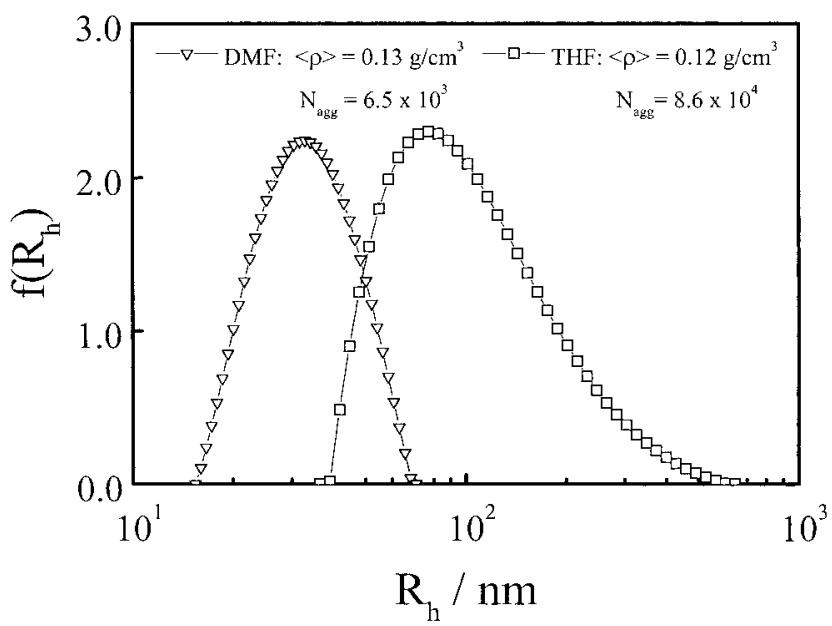

Figure 2. I nitial solvent effect on number distribution $f\left(R_{h}\right)$ of hydrodynamic radius of self-assembled Zn-MCPS-1.8K nanostructures formed by adding the polymer solution in THF in water, where initial polymer concentration was $1.00 \times 10^{-3}$ $\mathrm{g} / \mathrm{mL}$.

Table 1. Characterization of Monocarboxy-Terminated Polystyrene (MCPS) and Dicarboxy-Terminated Polystyrene (DCPS) Chains

\begin{tabular}{lccccr}
\hline \multicolumn{1}{c}{ sample } & $\begin{array}{c}\text { deg of } \\
\text { polymer }\end{array}$ & $\begin{array}{c}\mathrm{M}_{\mathrm{n}}\left(10^{3}\right. \\
\mathrm{g} / \mathrm{mol})\end{array}$ & $\mathrm{M}_{\mathrm{w}} / \mathrm{M}_{\mathrm{n}}$ & $\begin{array}{c}\mathrm{N}_{\text {ionic group }} \\
\text { chain }\end{array}$ & $\begin{array}{r}\mathrm{T}_{\mathrm{g}} \\
\left({ }^{\circ} \mathrm{C}\right)\end{array}$ \\
\hline MCPS-1.8K & 17 & 1.8 & 1.28 & 0.95 & 86 \\
MCPS-3.9K & 38 & 3.9 & 1.16 & 0.91 & 93 \\
MCPS-5.5K & 53 & 5.5 & 1.06 & 0.98 & 97 \\
MCPS-23.4K & 225 & 23.4 & 1.06 & 0.90 & 102 \\
DCPS-5.5K & 53 & 5.5 & 1.25 & 1.90 & 99
\end{tabular}

tion. Therefore, more polymer chains have a chance to be incorporated into one particle before they are collapsed and frozen. It is known that the intrachain contraction will lead to a density lower that the interchain association because the chains are not infinitely flexible. ${ }^{16,17}$ Such formed nanoparticles should be more close to a thermodynamic equilibrium. We did an insitu investigation of the association by monitoring the scattering intensity of the solution mixture during the addition of water. It showed that the association suddenly took place at a critical point (16 vol \% water) which is close to the $\theta$ composition of the THF/water mixture for polystyrene. For Na-MCPS-3.9K, adding the THF solution to water and adding water to the THF solution respectively led to $s_{\text {ionic }} \sim 5.7$ and $2.3 \mathrm{~nm}^{2}$. The difference in sionic indicates that kinetics played an important role in the self-assembly.

Figure 2 shows that if DMF instead of THF was used as the initial solvent to dissolve MCPS, smaller particles were formed with $\mathrm{N}_{\text {agg }} \sim 6.5 \times 10^{3}$ and $\langle\rho\rangle \sim 0.13 \mathrm{~g} / \mathrm{cm}^{3}$. It is known that DMF is a better solvent than THF for the ionic end group, and MCPS in DMF can exist as individual chains. In this case, we would expect that the interchain association is slower than the intrachain contraction in the microphase inversion so that a less

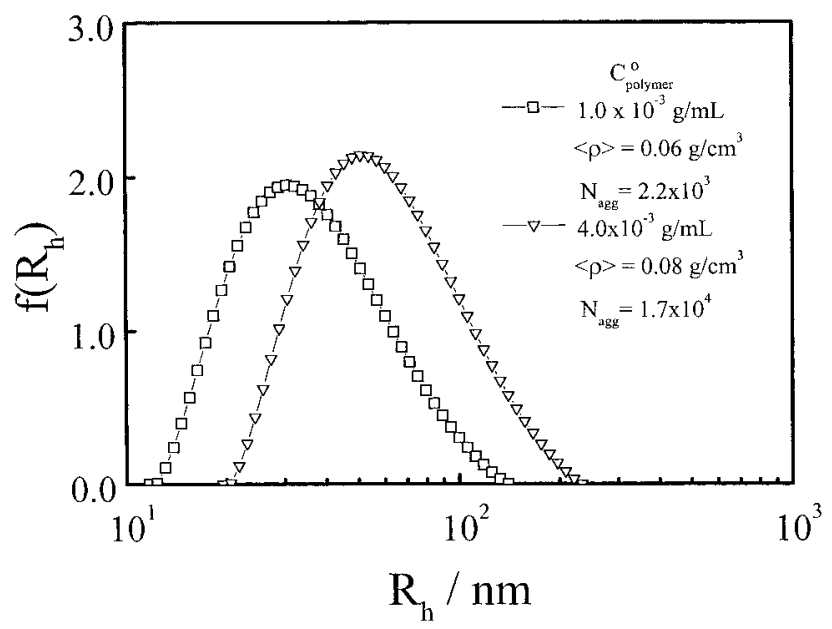

Figure 3. I nitial polymer concentration dependence of number distribution of hydrodynamic radius $f\left(R_{h}\right)$ of self-assembled Na-MCPS-1.8K nanostructures formed by adding the polymer solution in THF in water.

number of chains are self-assembled together. Naturally, if the initial polymer concentration in THF or DMF is lower, the interchain association would al so be suppressed so that smaller particles resulted, which is confirmed in Figure 3.

Table 2 summarizes the chain length dependence of the self-assembly. As expected, the particle size increases with the chain length. A larger particle with a larger surface area requires more ionic end groups to stabilize it. This is why both $\left\langle R_{h}\right\rangle$ and $N_{\text {agg }}$ increase with the chain length. As discussed before, we have sionic $=$ $\mathrm{S} / \mathrm{N}_{\text {agg }}=3 \mathrm{M}_{\mathrm{w}, \text { chain }} /(\langle\rho\rangle \mathrm{R})$, because $\mathrm{M}_{\mathrm{w} \text {, particle }}=\mathrm{M}_{\mathrm{w} \text {, chain }}$ $N_{\text {agg }}=4 \pi R^{3}\langle\rho\rangle / 3$ and $S=4 \pi R^{2}$. Table 2 shows that $S_{\text {ionic }}$ is nearly a constant in the range $4-6 \mathrm{~nm}^{2}$ when $\mathrm{Na}^{+}$ was used as counterion, and $\langle\rho\rangle$ increases with the chain length. Note that $\left\langle R_{h}\right\rangle$ is not proportional to $M_{w}$, chain because the polymer chains were collapsed. It can also be visualized that the self-assembly of longer ionomer chains with a relatively less ionic content requires more chains to be involved so that there are enough stabilizing ionic groups. It explains why $\langle\rho\rangle$ increases as the chain length, but sionic remains.

Table 2 also shows that for a given ionomer chain length the particle size varies when different types of counterions are used, in an increasing order of $\mathrm{Na}^{+}<$ $\mathrm{Li}^{+}<\mathrm{Zn}^{2+}$, corresponding to the increasing order of the dissociation constant of $\mathrm{COO}^{-} \mathrm{M}^{+}$; namely, a weak dissociation of $\mathrm{COO}^{-} \mathrm{M}^{+}$results in a weaker ionic repulsion so that each ionic end group can only stabilize a smaller surface area, so that $\mathrm{R}$ must be larger. Moreover, in comparison with MCPS, the self-assembly of DCPS led to much smaller nanostructures because one dicarboxyl group can stabilize a larger surface area than one monocarboxyl group. It is very interesting to note that the self-assembly of DCPS and MCPS results in a similar Sionic if we consider that one DCPS i onomer

Table 2. Summary of Static and Dynamic Laser Light Scattering Results of MCPS and DCPS Nanoparticles Formed by Adding the Ionomer THF Solution into Water, Where the Initial Ionomer Concentration Was $1 \times 10^{-3} \mathrm{~g} / \mathrm{mL}$

\begin{tabular}{cccccccc}
\hline sample & $\mathrm{M}_{\mathrm{w}, \text { particle }}(\mathrm{g} / \mathrm{mol})$ & $\left\langle\mathrm{R}_{\mathrm{h}}\right\rangle(\mathrm{nm})$ & $\left\langle\mathrm{R}_{\mathrm{g}}\right\rangle(\mathrm{nm})$ & $\left\langle\mathrm{R}_{\mathrm{g}}\right\rangle /\left\langle\mathrm{R}_{\mathrm{h}}\right\rangle$ & $\mathrm{N}_{\text {agg }}$ & $\mathrm{S}_{\text {ionic }}\left(\mathrm{nm}^{2}\right)$ & $\rho\left(\mathrm{g} / \mathrm{cm}^{3}\right)$ \\
\hline Na-MCPS-1.8K & $4.0 \times 10^{6}$ & 30 & 24 & 0.8 & $2.2 \times 10^{3}$ & 5.1 & 0.06 \\
Na-MCPS-3.9K & $9.8 \times 10^{6}$ & 34 & 31 & 0.9 & $2.5 \times 10^{3}$ & 5.7 & 0.10 \\
Na-MCPS-5.5K & $2.3 \times 10^{7}$ & 36 & 25 & 0.7 & $4.2 \times 10^{3}$ & 3.9 & 0.19 \\
Na-MCPS-23.4K & $2.4 \times 10^{8}$ & 69 & 67 & 1.0 & $1.0 \times 10^{4}$ & 5.8 & 0.29 \\
Na-DCPS-5.5K & $2.1 \times 10^{6}$ & 20 & 14 & 0.7 & $3.8 \times 10^{2}$ & 6.6 & 0.11 \\
Zn-MCPS-1.8K & $1.6 \times 10^{8}$ & 79 & 72 & 0.9 & $8.6 \times 10^{4}$ & 0.9 & 0.12 \\
Li-MCPS-3.9K & $2.5 \times 10^{7}$ & 43 & 34 & 0.8 & $6.4 \times 10^{3}$ & 3.6 \\
Zn-MCPS-3.9K & $1.7 \times 10^{9}$ & 176 & 158 & 0.9 & $4.2 \times 10^{4}$ & 0.9 & 0.12
\end{tabular}




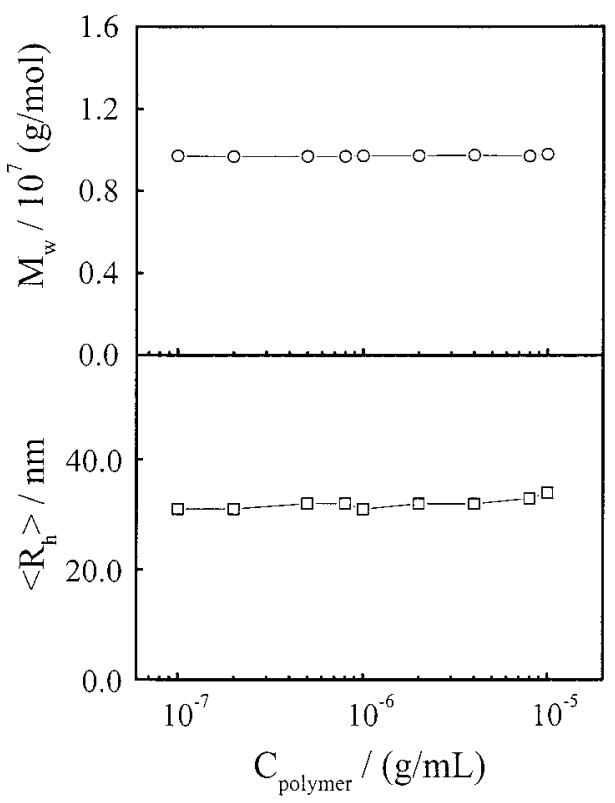

Figure 4. Effect of dilution on self-assembled Na-MCPS-3.9K nanostructures formed by adding the polymer solution in THF in water, where the dilution was done after the nanostructure formation.

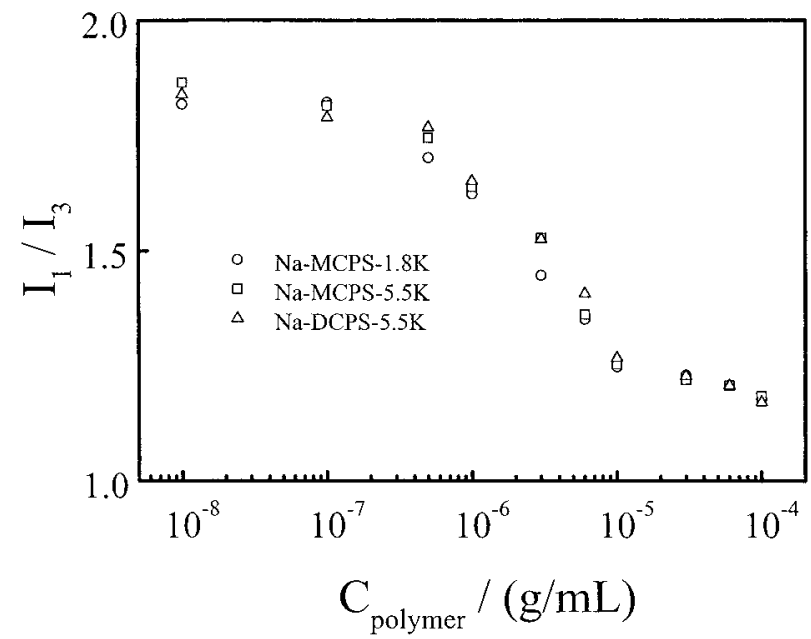

Figure 5. I onomer concentration dependence of fluorescence intensity ratio $\left(\mathrm{I}_{1} / \mathrm{I}_{3}\right)$ of first and third bands in pyrene emission spectrum, where pyrene concentration was kept at $2.0 \times 10^{-6}$ $\mathrm{M}$.

chain has two ionic groups. The values of $\left\langle R_{g}\right\rangle /\left\langle R_{h}\right\rangle$ listed in Table 2 are close to 0.8, a value predicted from uniform and nondraining spheres, indicating that the self-assembled micelle-like nanostructures are spherical and nearly uniform in density.

Figure 4 shows that in terms of $\left\langle R_{h}\right\rangle$ and $M_{w}$ the dilution has nearly no effect on the self-assembled nanostructures after they were formed in the microphase inversion, indicating that such formed metastable nanostructures are actually very stable even in an extremely dilute solution. This means either there is no dynamic equilibrium between the nanostructures and individual chains or its critical aggregation concentration (cac) is too low to be detected by laser light scattering. Figure 5 shows a typical polymer concentration dependence of the intensity ratio $\left(\mathrm{I}_{1} / \mathrm{I}_{3}\right)$ of the first and third bands in the pyrene emission spectrum. It is known that when the surroundings of pyrene change from polar to nonpolar, $\mathrm{I}_{1} / \mathrm{I}_{3}$ decreases, e.g., $\sim 1.8 \mathrm{in}$ water, $\sim 1.0$ in anionic surfactant micelles, and $\sim 0.6$ in cyclohexane. A comparison of Figures 4 and 5 shows that the decrease of $\mathrm{I}_{1} / \mathrm{I}_{3}$ from 1.9 to 1.2 as the polymer concentration increases is not related to the selfassembly. It actually indicates that most of the pyrene molecules are surrounded by water in the extremely dilute regime $\left(\mathrm{C}<1 \times 10^{-7} \mathrm{~g} / \mathrm{mL}\right.$ ). As the polymer concentration increases, more pyrene molecules are partitioned inside the hydrophobic particles, resulting in a decrease of $I_{1} / I_{3}$. The plateau val ue of $I_{1} / I_{3}$ at $C_{\text {polymer }}$ $>10^{-5} \mathrm{~g} / \mathrm{mL}$ is not as low as 0.95 in bulk polystyrene because each particle, on average, still contains $\sim 90 \%$ of water in its hydrodynamic volume. It is worth noting that the chain length and the type of ionic end group have nearly no effect on the polymer concentration dependence of $\mathrm{I}_{1} / \mathrm{I}_{3}$, further indicating that the decrease of $\mathrm{I}_{1} / \mathrm{I}_{3}$ is not related to the self-assembly.

In summary, MCPS and DCPS polymers with only one or two ionic groups at one chain end can undergo a self-assembly to form surfactant-free polymeric nanoparticles if the polymer solution in THF or DMF and water are mixed in a slow fashion. The self-assembly resembles the initial stage of surfactant-free emulsion polymerization. Our results showed that the self-assembly was controlled by a competition between intrachain association and interchain association. There exists a delicate balance between thermodynamics and kinetics. The size of the self-assembled micelle-like nanostructures is essentially governed by the surface area occupied per ionic end group. By varying experimental conditions, such as the initial ionomer concentration, solvent, the mixing sequence, and counterions, we can make either the intrachain contraction or the interchain association dominant so that the self-assembly can be tailored.

Acknowledgment. This work was supported by National Natural Science Foundation of China (NNSFC, No. 29974027, 29574154, 59773023), the National Basic Research Project-Macromolecular Condensed State, and the Research Grants Council of Hong Kong Special Administration Region Earmarked Grant 1999/2000 (CUHK4209/99P ,A/C2160122).

\section{References and Notes}

(1) Goodall, A. R.; Wilkinson, M. C.; Hearn, J. In Polymer Colloids; Fitch, R. M., Ed.; Plenum Press: New York, 1980.

(2) Tauer, K.; Kuhn, I. In Polymeric Dispersions: Principles and Applications; Asua, J. M., Ed.; Kluwer Academic Publishers: Dordrecht, The Netherlands, 1997; $p 49$.

(3) Goodall, A. R.; Wilkinson, M. C.; Hearn, J . J . Polym. Sci., Polym. Chem. Ed. 1977, 15, 2193.

(4) Chen, S.; Chang, H. J . Polym. Sci., Polym. Chem. Ed. 1985 23, 2615.

(5) Tauer, K.; Deckwer, R. Acta Polym. 1998, 49, 411.

(6) Kuhn, I.; Tauer, K. Macromolecules 1995, 28, 8112.

(7) Li, M.; Lu, L.; J iang, M. Macromol. Rapid Commun. 1995, $16,831$.

(8) Li, M.; J iang, M.; Zhu L.; Wu, C. Macromolecules 1997, 30, 2201.

(9) Li, M.; Zhang, Y.; J iang, M.; Wu, C. Macromolecules 1998, 31,1 .

(10) Li, M.; Wu, C.; J iang, M. J . Polym. Sci., Polym. Phys. Ed. 1997, 35, 1593.

(11) Zhong, X.; Eisenberg, A. Macromolecules 1994, 27, 1751.

(12) Morton, M.; Fetters, L. J . Rubber Chem. Technol. 1975, 48, 359.

(13) Mansson, P. J . Polym. Sci., Polym. Chem. Ed. 1980, 18, 1945

(14) Zhong, X.; Eisenberg, A. Macromolecules 1994, 27, 4914.

(15) J alal, N.; Duplessix, R. J . Phys. (Paris) 1988, 49, 1775.

(16) Wu, C.; Chan, K. K. J . Polym Sci., Polym. Phys. 1995, 33, 919.

(17) Wu, C.; Qian, R. Y. Qian, Napper, D. H. Macromol ecules 1995 28, 1592.

MA001120U 